\title{
Buffer-Aided Link Selection for Incremental Relaying Systems
}

\author{
Mostafa Darabi ${ }^{\dagger}$, Behrouz Maham ${ }^{\dagger}$ and Yan Zhang ${ }^{\ddagger}$ \\ ${ }^{\dagger}$ School of ECE, College of Engineering, University of Tehran, Iran \\ $\ddagger$ Simula Research Laboratory, University of Oslo, Norway \\ emails: \{mostafa.darabi, bmaham\}@ut.ac.ir, yanzhang@simula.no
}

\begin{abstract}
In this paper, we consider a three-node wireless network comprising of a source and two users. Both users need to decode the transmitted data correctly. User 1 has better position to the source than user 2 most of time slots. User 1 has buffer to store the transmitted information by the source. Thus, in the case of wrong decoding at user 2 , user 1 can resend data to user 2 some time slots later. In this paper, we propose a novel incremental relaying based adaptive link selection policy that exploits incremental relaying and buffer to maximize the throughput of the network. That is, based on the channel quality of the available links, each time slot is allocated either to the source or user 1 to transmit data. Both delay constrained and delay tolerant transmission schemes are studied. We model the variation of the buffer at user 1 as a Markov Chain and calculate the outage probability of the proposed policy. Our simulation results show that the proposed scheme achieves higher throughput and lower outage probability compared to the recently proposed link selection policies with or without buffer.

Index Terms-Buffer-aided relay channel, incremental relaying, adaptive link selection, throughput maximization, outage probability, Markov Chain.
\end{abstract}

\section{INTRODUCTION}

The three-node relay network was first investigated by van der Meulen in [1]. Later, Host-Madsen and Zhang in [2] calculated the capacity of a network consisting of a source, a half-duplex decode-and-forward relay and a destination. Their work is based on the existence of predetermined schedule for data transmission and reception in the network. Since then, many of papers have been published in the area of threenode relay channel. However, only few of them have assumed the existence of the buffer in the relay (see, e.g., [3], [4]). In [3], the relay exploits buffer to receive information for a fixed number of time slots before forwarding them to the destination. In [4], the relays are equipped with buffers and the relay selection is used to transmit data. More specifically, buffers are used to select the relay with best source-relay link for reception and the relay with best relay-destination for transmission. Later in [5], Zlatanov et al. introduced a bufferaided adaptive link selection scheme, in which the relay has buffer in order to decide freely when to receive and when to transmit the data packets. In [5], direct link between the source and destination is not available and packets can be transmitted only through the middle relay. It is proved in [5] that the buffer-aided adaptive link selection achieves higher throughput compared to the conventional relaying policy without buffer.

In [6], incremental relaying is exploited in a simple threenode network with available direct link between the source

This work was supported by the Iran National Science Foundation (INSF) through the project 91000529 . and the destination. By using incremental relaying, degrees of freedom of the network are used more efficiently. Moreover, it has been shown that the diversity order of the network is equal to two in the Rayleigh fading channel.

In this paper, we consider a simple three-node relay network with one source and two users. User 1 has buffer and in the case of wrong decoding at user 2, user 1 acts as a half-duplex decode-and-forward relay for user 2 . We assume that full CSI are available at the transmitters, thus, the source and user 1 can use capacity-achieving codes for the data transmission. Furthermore, incremental relaying is exploited to efficiently improve the throughput of the proposed link selection policy. We propose a buffer-aided incremental relaying based adaptive link selection, where in each time slot, based on the channel quality and status of the buffer, either the source or user 1 is selected for data transmission. We consider both delay limited and delay tolerant transmissions. For the delay tolerant case, we derive the optimum average throughput. For the delay limited case, we limit the queue size by forcing user 1 to transmit if its buffer gets full. Based on our simulation results, the proposed buffer-aided incremental relaying based adaptive link selection has significant throughput improvement compared to the other recently proposed buffer-aided link selection policies in [5].

We investigate the outage probability of the proposed policy when capacity-achieving code is not exploited at the transmitters. We model the evolution of the buffer of user 1 as Markov Chain. Thus, the outage probability of the network can be found easily by using the state transition matrix and the steady state of the Markov Chain. According to the simulation results, our proposed policy has a diversity gain of two for high buffer size.

\section{SySTEM MODEL}

A three-node wireless network is shown in Fig. 1. In this figure, the source $S$, sends data packets to two users $U_{1}$ and $U_{2}$ simultaneously. If an error occurs during decoding of the received data at user 2 , user 1 acts as a half-duplex decodeand-forward relay for user 2 . In this paper, user 1 has buffer to avoid data loss and to decide optimally when to receive and when to transmit information to user 2. At user 1, the received codewords are decoded and stored in the buffer to be forwarded later in the case of wrong decoding at user 2. The size of the buffer of user 1 is denoted by $L$. At user 2, ACK or NACK is transmitted back over separate narrow band error free feedback channel to make the network aware of the packet reception status. For simplicity, in this paper, the number of transmitted bits is normalized by the number of 
symbols in each time slot, i.e., the number of bits is divided by the number of symbols in a codeword. The normalized number of data packets in bits/symbol in the buffer of user 1 at the end of $i$-th time slot, is represented by $Q(i)$. At the initial time slot, because of the empty buffer, $Q(i)$ is equal to zero. On the other hand, $Q(i)$ varies by one in the case of successful data transmission or reception at user 1 . All wireless links undergo zero mean Additive White Gaussian Noise (AWGN) with unit variance and frequency non selective Rayleigh block fading. Thus, during one time slot, the fading coefficients are fixed but vary independently from one time slot to another. The source and user 1 transmit with the fixed powers of $P_{S}$ and $P_{U_{1}}$, respectively. The variables $H_{S U_{1}}(i), H_{S U_{2}}(i)$ and $H_{U_{1} U_{2}}(i)$ represent the squared channel gains of the $S-U_{1}$, $S-U_{2}$ and $U_{1}-U_{2}$ links in the $i$-th time slot, respectively. The instantaneous link signal-to-noise ratios (SNRs) of the $S-U_{1}$, $S-U_{2}$ and $U_{1}-U_{2}$ links in the $i$-th time slot, are denoted by $S U_{1}(i)=H_{S U_{1}}(i) P_{S}, S U_{2}(i)=H_{S U_{2}}(i) P_{S}$ and $U_{1} U_{2}(i)=$ $H_{U_{1} U_{2}}(i) P_{U_{1}}$, respectively. The average link SNRs of the $S-$ $U_{1}, S-U_{2}$ and $U_{1}-U_{2}$ links are denoted by $\gamma_{S U_{1}}, \gamma_{S U_{2}}$ and $\gamma_{U_{1} U_{2}}$, respectively. Channel gains are assumed mutually independent, ergodic and stationary random processes. The instantaneous capacities of the links $S-U_{1}, S-U_{2}$ and $U_{1}-U_{2}$ are denoted by $C_{S U_{1}}(i), C_{S U_{2}}(i)$ and $C_{U_{1} U_{2}}(i)$, respectively.

Due to the availability of CSI at the transmitters, the source and user 1 exploit capacity-achieving codes. Because the buffer exists only at user 1 and in order to avoid data loss, the source transmits with rate of $C_{S U_{1}}(i)$. Also, user 1 transmits to user 2 with the rate of instantaneous capacity of the $U_{1}-U_{2}$ link. Thus, the source transmits with the following rate

$$
R_{S U_{1}}(i)=\log _{2}\left(1+S U_{1}(i)\right)=C_{S U_{1}}(i) .
$$

User 2 receives data with the rate of $R_{S U_{1}}(i)$ too. The transmission rate of the source may not be equal to $C_{S U_{2}}(i)$ and therefore, the data transmission in the $S-U_{2}$ link may be incorrect. In the case of wrong decoding at user 2, data packets are stored in the buffer of user 1 to be retransmitted later. Thus, In the case of wrong decoding at user 2, the number of normalized data packets in the buffer at the end of $i$-th time slot is

$$
Q(i)=Q(i-1)+R_{S U_{1}}(i) .
$$

On the other hand if at user 2 , the received data is decoded successfully, data is not stored in the buffer of user 1 and the amount of data elements in the buffer is not changed. If user 1 is selected for the data transmission in the $i$-th time slot, the number of normalized data packets which are transmitted is limited by the number of normalized data packets in its buffer and the instantaneous capacity of the $U_{1}-U_{2}$ link. Thus, user 1 transmits to user 2 with the rate of

$$
\begin{aligned}
R_{U_{1} U_{2}}(i) & =\min \left(Q(i-1), \log _{2}\left(1+U_{1} U_{2}(i)\right)\right) \\
& =\min \left(Q(i-1), C_{U_{1} U_{2}}(i)\right) .
\end{aligned}
$$

Therefore, the number of normalized data packets in the buffer at the end of $i$-th time slot is

$$
Q(i)=Q(i-1)-R_{U_{1} U_{2}}(i) .
$$

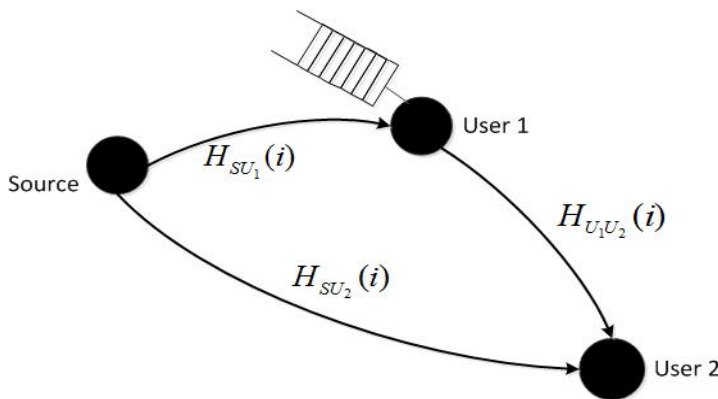

Fig. 1. The wireless three-node network in which user 1 has buffer.

In Section III, we assume that global CSI and buffer state information can be achieved at user 1 by appropriate signaling. Thus, user 1 can coordinate the transmission of data in the network. To do this, in each time slot, user 1 determines the transmitting link among the available links through an errorfree channel.

\section{Proposed Policy}

In the early literature on the three-node communication channels, time schedule for the data transmission of the nodes are predetermined before the starting of the data transmission. In this paper, by using buffer in the network, the source and user 1 decide freely in which time slot to transmit and in which time slot to be silent. We propose a new relaying scheme exploiting incremental relaying based adaptive link selection. That is, in each time slot based on the channel and buffer state information, the time slot is allocated either to the source or user 1 for the data transmission. If the source is selected for the data transmission, it transmits new data packet to both users. At user 2, it is investigated whether the received packet is decoded correctly or not. The one-time Automatic RepeatreQuest (ARQ) is used at user 2 to make the network aware of the packets reception status. If at user 2, the received data packet is decoded wrongly, NACK is sent back and related packet is stored in the buffer of user 1 to be retransmitted later. On the other hand if at user 2, the received data is decoded correctly, the corresponding data packet is not stored in the buffer.

Furthermore, if user 1 is selected for the data transmission, it retransmits the data packet from its buffer. Because of using capacity-achieving code, after the data retransmission, it is removed from the buffer. It is worth noting that user 1 can receive data from the source when the buffer is not full and the $S-U_{1}$ link is not in outage. User 2 can receive data from user 1 when the buffer of user 1 is not empty and the $U_{1}-U_{2}$ link is not in outage.

\section{A. Buffer-Aided Link Selection for Delay Tolerable Incremen- tal Relaying Systems}

In this section, we assume that the buffer of user 1 has infinite size and the transmission powers of the source and user 1 are fixed. Under these assumptions, we investigate the incremental relaying based adaptive link selection scheme and obtain the maximum throughput. In the next subsection, the policy with limited size buffer will be discussed. 
We use the binary variable of $a_{i}$ to indicate that whether the data transmission between the source and user 2 is successful or not. If the instantaneous capacity of $S-U_{2}$ link is greater than or equal to the instantaneous capacity of $S-U_{1}$ link, i.e., user 2 can decode the received data packets from source correctly, we set $a_{i}=1$, otherwise $a_{i}$ is set to zero. We exploit $b_{i} \in\{0,1\}$ as a binary decision variable, to indicate that whether the source or user 1 transmits in the $i$-th time slot. The variable $b_{i}$ is set to zero to point out that the source transmits in the $i$-th time slot. On the other hand, if user 1 transmits in $i$-th time slot, we set $b_{i}=1$. For the nonnegative, stationary and ergodic SNRs, if $N$ goes to infinity, the source to user 1, the source to user 2 and user 1 to user 2 achievable average transmission rates, denoted by $\bar{R}_{S U_{1}}, \bar{R}_{S U_{2}}$ and $\bar{R}_{U_{1} U_{2}}$, respectively, can be written as

$$
\bar{R}_{S U_{1}}=\lim _{N \rightarrow \infty} \frac{1}{N} \sum_{i=1}^{N}\left(1-b_{i}\right) C_{S U_{1}}(i)=\mathbb{E}\left\{(1-b) C_{S U_{1}}\right\}
$$

$$
\bar{R}_{S U_{2}}=\lim _{N \rightarrow \infty} \frac{1}{N} \sum_{i=1}^{N} a_{i}\left(1-b_{i}\right) C_{S U_{1}}(i)=\mathbb{E}\left\{a(1-b) C_{S U_{1}}\right\},
$$

$$
\begin{aligned}
\bar{R}_{U_{1} U_{2}} & =\lim _{N \rightarrow \infty} \frac{1}{N} \sum_{i=1}^{N} b_{i} \min \left\{Q(i-1), C_{U_{1} U_{2}}(i)\right\} \\
& =\mathbb{E}\left\{b \min \left\{Q, C_{U_{1} U_{2}}\right\}\right\} .
\end{aligned}
$$

For notational simplicity, the time index $i$ in the expectations is dropped. In addition, the achievable average reception rate of both users which are denoted by $\bar{R}_{U_{1}}$ and $\bar{R}_{U_{2}}$, can be written as

$$
\begin{aligned}
& \bar{R}_{U_{1}}=\bar{R}_{S U_{1}}, \\
& \bar{R}_{U_{2}}=\bar{R}_{S U_{2}}+\bar{R}_{U_{1} U_{2}} .
\end{aligned}
$$

According to the system model in Fig. 1, every data packet which is received by user 2 , has been received by user 1 simultaneously or in the earlier time slot. Thus, $\bar{R}_{U_{2}}$ is lower than or equal to $\bar{R}_{U_{1}}$. Therefore, by maximizing $\bar{R}_{U_{2}}$, the throughput of both users and the total average throughput of the network are maximized. By using $a_{i}$ and $b_{i}, \bar{R}_{U_{2}}$ can be written as

$$
\begin{aligned}
\bar{R}_{U_{2}} & =\mathbb{E}\left\{a(1-b) C_{S U_{1}}\right\}+\mathbb{E}\left\{b \min \left\{Q, C_{U_{1} U_{2}}\right\}\right\} \\
& =\mathbb{E}\{a\} \bar{R}_{S U_{1}}+\bar{R}_{U_{1} U_{2}} .
\end{aligned}
$$

Now, we want to maximize the average reception rate of user 2 by optimizing the link utilization. More specifically, $\bar{R}_{U_{2}}$ is maximized by finding the optimal sequence of $b_{i}$.

Theorem 1. Assume that the channel gains and link SNRs are non-negative, ergodic and stationary random processes. The maximum throughput for the network is obtained when the queue at the buffer of user 1 is at the edge of non-absorbtion, i.e., $\bar{R}_{S U_{1}}-\bar{R}_{S U_{2}}=\bar{R}_{U_{1} U_{2}}$ and the below necessary condition is satisfied.

$$
\mathbb{E}\left\{(1-a)(1-b) C_{S U_{1}}\right\}=\mathbb{E}\left\{b C_{U_{1} U_{2}}\right\} .
$$

In addition, $\bar{R}_{U_{2}}$ can be written as

$$
\bar{R}_{U_{2}}=\mathbb{E}\left\{a(1-b) C_{S U_{1}}\right\}+\mathbb{E}\left\{b C_{U_{1} U_{2}}\right\} .
$$

Proof. Please refer to Appendix A.

The optimal sequence of $b_{i}$ has to satisfy (11). Hence, we should solve the following optimization problem

$$
\begin{gathered}
\max _{b_{i}} \quad \lim _{N \rightarrow \infty} \frac{1}{N} \sum_{i=1}^{N}\left\{a_{i}\left(1-b_{i}\right) C_{S U_{1}}(i)+b_{i} C_{U_{1} U_{2}}(i)\right\} \\
\text { S.t } \quad \text { C1: } \lim _{N \rightarrow \infty} \frac{1}{N} \sum_{i=1}^{N}\left(1-a_{i}\right)\left(1-b_{i}\right) \log _{2}\left(1+S U_{1}(i)\right) \\
=\lim _{N \rightarrow \infty} \frac{1}{N} \sum_{i=1}^{N} b_{i} \log _{2}\left(1+U_{1} U_{2}(i)\right) \\
\text { C2: } b_{i}\left(1-b_{i}\right)=0 \quad \forall i
\end{gathered}
$$

where constraint $\mathrm{C}_{1}$ is due to the theorem 1 and constraint $\mathrm{C}_{2}$ states that the sequence $b_{i}$ consists of binary numbers.

Theorem 2. The optimal sequence of $b_{i}$ that maximizes the total throughput of the network is as below:

$$
b_{i}= \begin{cases}1 & \text { if } \log _{2}\left(1+U_{1} U_{2}(i)\right) \geq \lambda \log _{2}\left(1+S U_{1}(i)\right) \\ 0 & \text { otherwise }\end{cases}
$$

The parameter $\lambda$ is decision threshold and should satisfy (11).

Proof. Please refer to Appendix B.

From theorem 2, It can be shown that at the $i$-th time slot the optimal sequence of $b_{i}$, depends only on the $i$-th instantaneous link SNRs. Thus, our proposed scheme can be implemented easily.

\section{B. Buffer-Aided Link Selection for Delay Limited Incremental} Relaying Systems

Before this, we have ignored delay limitation and have considered buffer with infinite size at user 1 . But most of the time, there could be some limits on the delay and on the size of the buffer in the network. In this subsection, the size of the buffer at user 1 is $L$ and we assume delay constrained scheme and modify the proposed policy under this limitation. Again, we assume that both the source and user 1 transmit with fixed power of $P$ at all the time slots. The proposed policy is given in table 1 .

Throughout the reminder of the paper, the number of the data packets which are stored at the buffer of user 1 is shown by $\eta(Q)$. In order to obtain the outage probability of the proposed scheme, we model the system as a Markov Chain. That is, the number of data packets at the buffer determines the state of the MC. Thus, the $i$-th state of the MC is denoted by $s_{i}=(\eta(Q))$ with assumption of $1 \leq i \leq(L+1)$. In the next section, the outage probability of our proposed scheme is computed by using the MC model. 
TABLE I

BUFFER-AIDED LINK SELECTION FOR DELAY LIMITED INCREMENTAL RELAYING SYSTEMS

(1) If the buffer of user 1 is not empty and it has enough space for the incoming data or equivalently the below condition is fulfilled, $b_{i}$ is selected based on (14).

$0<Q(i-1) \leq L-\log _{2}\left(1+S U_{1}(i)\right)$.

(2) If the buffer of user 1 is not empty and the instantaneous capacity of $S-U_{2}$ link is greater than or equal to the instantaneous capacity of $S-U_{1}$ link or equivalently the below condition is fulfilled, $b_{i}$ is selected based on (14). In this case, since user 2 can decode received data correctly, data is not stored in the buffer of user 1 .

$0<Q(i-1)$ and $C_{S U_{1}}(i) \leq C_{S U_{2}}(i)$

(3) If the buffer of user 1 does not have enough space for the incoming data and the instantaneous capacity of $S-U_{2}$ link is lower than the instantaneous capacity of $S-U_{1}$ link or equivalently the below condition is satisfied, we set $b_{i}=1$.

$L-\log _{2}\left(1+S U_{1}(i)\right)<Q(i-1)$. and $C_{S U_{2}}(i)<C_{S U_{1}}(i)$

(4) If $Q(i-1)=0$, we set $b_{i}=0$.

\section{Outage Probability Analysis}

In this section, we assume that capacity-achieving codes are not used in the network and the source transmits with fixed transmission rate of $r_{0}$ in bits per channel use (BPCU). Hence, the outage may occur if the capacity of one link is lower than the instantaneous data transmission rate through the link. To calculate the outage probability of the proposed scheme, we exploit the theoretical framework of [8]. Our contribution compared to [8] is that we have additional ways of data transmission through the $S-U_{2}$ link. In order to calculate the outage probability of the proposed policy, at first the state transition matrix is constructed. The state transition matrix shows the connection between the states of the MC with their probabilities. Let $\mathbf{T}$ denotes the state transition matrix with size of $(L+1) \times(L+1)$. In this matrix, the entry $\mathbf{T}_{(i, j)}=\operatorname{Pr}\left(s_{i} \rightarrow s_{j}\right)=\operatorname{Pr}\left(X_{t+1}=s_{j} \mid X_{t}=s_{i}\right)$ indicates the transition probability to move from state $s_{i}$ to state $s_{j}$ in two consecutive time slots. The number of participating links in the proposed policy and the number of data packets in the buffer determine the transition probabilities between the states of the MC. For example, when the buffer is full, user 1 can not receive data and only the $U_{1}-U_{2}$ link can participate in the link selection policy. On the other hand, if the buffer of user 1 is empty, user 1 can only receive the data and only $S-U_{1}$ and $S-U_{2}$ links can be selected. In other cases, user 1 can receive or send data and thus all links are available in the proposed scheme. As a result, for the $i$-th state of the Markov Chain, there are $n_{i}$ available links in the proposed policy.

$$
n_{i}= \begin{cases}2 & \text { if } 0<\eta(Q)<L, \\ 1 & \text { elsewhere. }\end{cases}
$$

In each time slot, the queue size of user 1 is changed according to the following rule: (I) If user 1 is selected to transmit and data packet is received correctly at user 2, the queue size is decreased by one. (II) If user 1 is selected to transmit and data packet is decoded wrongly at user 2 , the queue size is not changed. (III) If the data transmission from the source to user 1 and user 2 are unsuccessful and error free, respectively, data is not stored in the buffer. Thus, the amount of data elements in the buffer is not changed. (IV) If an outage occurs in the source transmission to both users, the queue size of user 1 remains unchanged. (V) If user 1 is selected to receive data and information is received correctly at both users, number of data packets in buffer of user 1 is not changed. (VI) If the source transmits data and user 1 decodes it successfully but user 2 decodes it wrongly, the queue size of user 1 is increased by one. We can show the connectivity between the states of the system, by defining two sets of $A_{i}^{-}$ and $A_{i}^{+}$for the $i$-th state. The set $A_{i}^{-}$consists of all the states that are connected to the $s_{i}$ state which have $i-1$ data elements in the buffer of user 1 . The set $A_{i}^{+}$is comprised of all the states that are connected to the $s_{i}$ state which have $i+1$ data elements in the buffer of user 1 .

$$
\begin{aligned}
& A_{i}^{-}=\left\{\bigcup_{1 \leq j \leq L+1} s_{j}: s_{j}-s_{i}=-1\right\}, \\
& A_{i}^{+}=\left\{\bigcup_{1 \leq j \leq L+1} s_{j}: s_{j}-s_{i}=+1\right\} .
\end{aligned}
$$

Transition from one state to another one requires both the selection of the related link by the proposed policy and the successful data transmission in that link. Channel links in the network are i.i.d. and symmetric. Thus, at the state $s_{i}$, a link is selected among the $n_{i}$ available links with the probability of $\frac{1}{n_{i}}$. The probability of moving from state $s_{i}$ to one of the states in $A_{i}^{-}$and $A_{i}^{+}$are denoted by $\operatorname{Pr}_{i}^{-}$and $\operatorname{Pr}_{i}^{+}$, respectively. These probabilities can be calculated by using statistics as follows

$\operatorname{Pr}_{i}^{-}=\frac{1}{n_{i}}\left[1-\left(1-\exp \left(-\frac{2^{r_{0}}-1}{P}\right)\right)^{n_{i}}\right]$,

$\operatorname{Pr}_{i}^{+}=\frac{1}{n_{i}}\left[1-\left(1-\exp \left(-\frac{2^{r_{0}}-1}{P}\right)\right)^{n_{i}}\right]\left(1-\exp \left(-\frac{2^{r_{0}}-1}{P}\right)\right)$.

Therefore, at the state $s_{i}$, the probability of staying and leaving the state, denoted by $\operatorname{Pr}_{i}^{\text {stay }}$ and $P r_{i}^{\text {leave }}$ respectively, can be written as

$$
\begin{gathered}
\operatorname{Pr}_{i}^{\text {leave }}=P r_{i}^{-}+P r_{i}^{+}, \\
\operatorname{Pr}_{i}^{\text {stay }}=1-P r_{i}^{\text {leave }} .
\end{gathered}
$$

Thus, for $i, j \in\{1,2, \ldots, L+1\}$, the state transition matrix can be written as

$$
\mathbf{T}_{(i, j)}= \begin{cases}P r_{i}^{\text {stay }} & \text { if } j=i \\ P r_{i}^{+} & \text {if } S_{j} \in A^{+} \\ P r_{i}^{-} & \text {if } S_{j} \in A^{-}\end{cases}
$$

The stationary distribution of the Markov Chain is denoted by $\mathbf{S}$. we have

$$
\mathbf{T S}=\mathbf{S}
$$

and

$$
\mathbf{B S}=\mathbf{b},
$$

in which $\mathbf{b}=(11 \ldots 1)^{T}$ and $\mathbf{B}_{i, j}=1, \forall i, j$. By adding (23) and (24) and with some mathematical manipulations, we reach to

$$
\mathbf{S}=(\mathbf{T}-\mathbf{I}+\mathbf{B})^{-1} \mathbf{b} .
$$




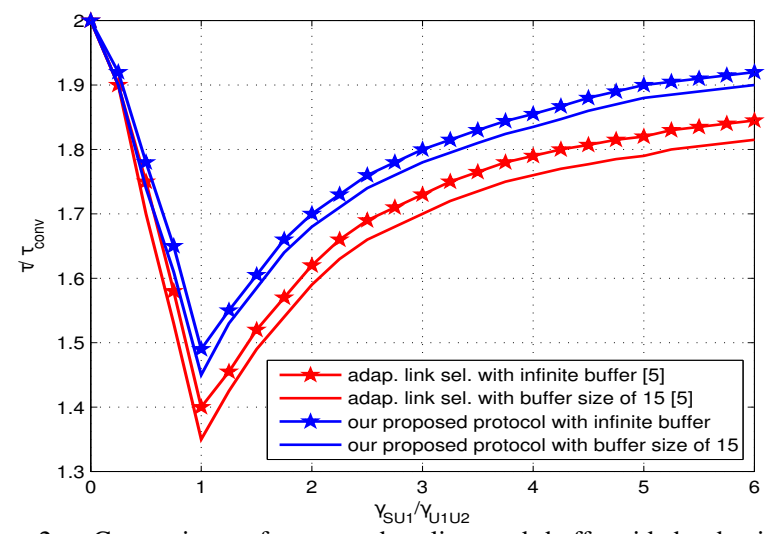

Fig. 2. Comparison of proposed policy and buffer-aided adaptive link selection which is introduced in [5] in terms of $\frac{\text { throughput }_{\max }}{\text { throughput }_{\text {conv }}}$.

The probability that the buffer at user 1 is unchanged, which is denoted by $P r_{\text {fixed }}$, can be calculated as

$$
\operatorname{Pr}_{\text {fixed }}=\sum_{i=1}^{L+1} \mathbf{S}_{i} \operatorname{Pr}_{i}^{\text {stay }}=\operatorname{diag}(\mathbf{T}) \mathbf{S} .
$$

Among the six cases of the rule of queue changing in the previous page, an outage occurs in the cases of (II), (III), (IV) and (VI). In order to calculate the outage probability, we should subtract the probability of case (V) from the probability of unchanged buffer and add the probability of case (VI) to it. The probability of cases $(\mathrm{V})$ and $(\mathrm{VI})$ which are denoted by $\operatorname{Pr}_{5}$ and $\operatorname{Pr}_{6}$ respectively, are as follows

$$
\begin{aligned}
\operatorname{Pr}_{5}=\sum_{i=1}^{L+1} \mathbf{S}_{i} \frac{1}{n_{i}} & {\left[1-\left(1-\exp \left(-\frac{2^{r_{0}}-1}{P}\right)\right)^{n_{i}}\right] } \\
& \exp \left(-\frac{2^{r_{0}}-1}{P}\right), \\
\operatorname{Pr}_{6}=\sum_{i=1}^{L+1} \mathbf{S}_{i} \frac{1}{n_{i}} & {\left[1-\left(1-\exp \left(-\frac{2^{r_{0}}-1}{P}\right)\right)^{n_{i}}\right] } \\
& \left(1-\exp \left(-\frac{2^{r_{0}}-1}{P}\right)\right) .
\end{aligned}
$$

Hence, the outage probability of our proposed incremental relaying based adaptive link selection scheme can be written as

$$
P_{\text {out }}=\operatorname{diag}(\mathbf{T}) \mathbf{S}-\operatorname{Pr}_{5}+P r_{6} .
$$

\section{Simulation Result}

In this section, we compare the throughput of our suggested policy with the throughput of buffer-aided adaptive link selection scheme in [5]. In all simulations, we consider Rayleigh block fading. In the first simulation, we assume buffer with infinite size and also buffer with size of 15 packets and investigate the achievable throughput with fixed transmission powers at the source and user 1 . We use the throughput of the conventional relaying without buffer for comparison purpose.

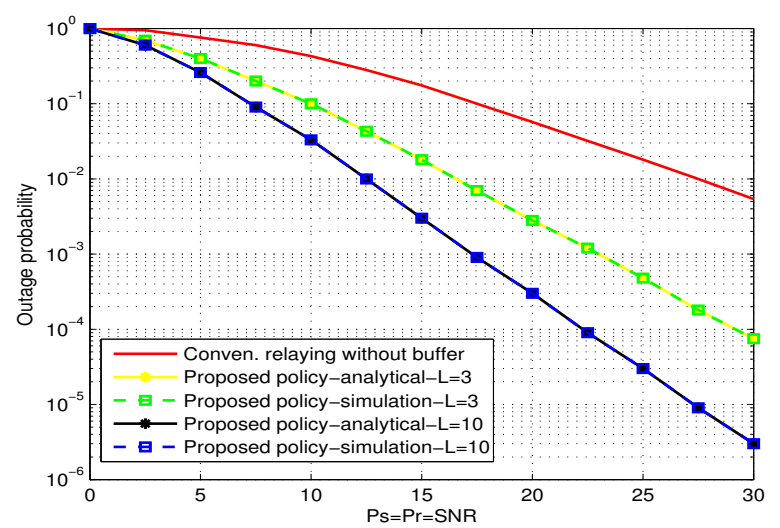

Fig. 3. The outage probability performance versus the SNR $[\mathrm{dB}]$.

In Fig. 2, we show the throughput of our proposed policy and the policy of buffer-aided adaptive link selection which is introduced in [5] as a function of $\frac{\gamma_{U_{1} U_{2}}}{\gamma_{S U_{1}}}$ with assumption of $\gamma_{S U_{1}}=1$. From Fig. 2, it is clear that our suggested policy outperforms buffer-aided adaptive link selection in terms of the throughput. Also the throughput is minimized where $\gamma_{U_{1} U_{2}}$ is equal to $\gamma_{S U_{1}}$. Due to equality of link's SNR in this point, conventional and suggested relaying schemes are equivalent. Thus, Fig. 2 is valley shaped. In addition, in Fig. 2, by increasing the buffer size, the throughput is increased.

In Fig. 3, we plot the outage probability in terms of the link SNRs with assumption of 1 bit per channel use source data rate. We calculate outage probability for two cases, when $L=3$ and when $L=10$. As can be seen from this figure, our analytical and simulation values of outage probability is close enough to each other and by increasing the buffer size, diversity gain of two is achieved. Also our simulation results show that the proposed link selection scheme achieves lower outage probability compared to conventional half-duplex relaying without buffer.

\section{CONCLUSION}

In this paper, we have proposed a novel adaptive link selection policy with incremental relaying to further increase the throughput. For both cases of the delay tolerant and the delay constraint networks, we maximized the throughput in terms of the available links for fixed transmission power of the source and user 1 . We showed that our suggested policy outperforms buffer-aided adaptive link selection policy which is introduced in [5], in terms of average throughput. When there is no CSI at the transmitters, we modeled the evaluation of the buffer of user 1 as a Markov Chain and then computed the outage probability of the proposed scheme. There are some possible extensions for this paper, including the investigation of the effect of imperfect channel knowledge on the performance of the network, using some network coding to increase further the throughput, power control methods and delay analysis.

\section{APPENDIX A}

Throughout the proof, $N$ goes to infinity and the cardinality of a set is denoted by |.|. Due to the conservation of the flow, 
the arrival rate into the buffer of user 1 is greater than or equal to the departure rate out of the buffer, i.e.,

$$
\bar{R}_{S U_{1}}-\bar{R}_{S U_{2}} \geq \bar{R}_{U_{1} U_{2}} .
$$

We will show that in the optimal policy $\bar{R}_{S U_{1}}-\bar{R}_{S U_{2}}=$ $\bar{R}_{U_{1} U_{2}}$. To show this, let $I_{1}, I_{2}$ and $I_{3}$ be the sets comprising of time slots $i$ in which $b_{i}=0, a_{i}, b_{i}=0$ and $b_{i}=1$, respectively. $I_{2}$ is subset of $I_{1}$ and $I_{1}$ and $I_{3}$ are two disjoint sets. If $\bar{R}_{S U_{1}}-\bar{R}_{S U_{2}}>\bar{R}_{U_{1} U_{2}}$, we can move some indices $i$ from $I_{1}$ to $I_{3}$ and thereby increase $\bar{R}_{U_{1} U_{2}}$ and decrease $\bar{R}_{S U_{1}}$. According to (10), $\bar{R}_{U_{2}}$ is equal to $\mathbb{E}\{a\} \bar{R}_{S U_{1}}+\bar{R}_{U_{1} U_{2}}$. Then, because of $\mathbb{E}\{a\} \leq 1$, by increasing $\bar{R}_{U_{1} U_{2}}$ and decreasing $\bar{R}_{S U_{1}}$ the average reception rate of user 2 is increased. Therefore, $\bar{R}_{S U_{1}}-\bar{R}_{S U_{2}}>\bar{R}_{U_{1} U_{2}}$ can not hold and in the optimal policy $\bar{R}_{S U_{1}}-\bar{R}_{S U_{2}}=\bar{R}_{U_{1} U_{2}}$. Now, we want to check wether the following equation is correct or not

$$
\frac{1}{N} \sum_{i \in I_{3}} C_{U_{1} U_{2}}(i)>\bar{R}_{U_{1} U_{2}} .
$$

Since in the optimal policy, the buffer of user 1 is at the edge of non-absorbtion, we can write

$$
\begin{aligned}
& \bar{R}_{U_{1} U_{2}}=\frac{1}{N} \sum_{i \in I_{3}} \min \left\{Q(i-1), C_{U_{1} U_{2}}(i)\right\} \\
& =\bar{R}_{S U_{1}}-\bar{R}_{S U_{2}}=\frac{1-\mathbb{E}\{a\}}{N} \sum_{i \in I_{1}} C_{S U_{1}}(i) .
\end{aligned}
$$

Assume that $\xi$ is a small subset of $I_{3}$, i.e., $\frac{|\xi|}{N} \rightarrow 0$. Then, by moving subset of $\xi$ from $I_{3}$ to $I_{1}$, the arrival rate into the buffer will be greater than the departure rate out of the buffer, i.e., $\bar{R}_{S U_{1}}(1-\mathbb{E}\{a\})>\bar{R}_{U_{1} U_{2}}$. Therefore, the normalized number of data elements stored in the buffer exceeds the number of departure bits, i.e., $C_{U_{1} U_{2}}(i) \leq Q(i-1)$. Thus, according to (7) we have

$$
\begin{aligned}
\frac{1}{N} \sum_{i \in I_{3} \backslash \xi} C_{U_{1} U_{2}}(i) & =\bar{R}_{U_{1} U_{2}}<(1-\mathbb{E}\{a\}) \bar{R}_{S U_{1}} \\
& =\frac{1-\mathbb{E}\{a\}}{N} \sum_{i \in I_{1} \bigcup \xi} C_{S U_{1}}(i) .
\end{aligned}
$$

Based on (31), (32) and (33), for the subset $\xi$ we should have

$$
\begin{array}{r}
\frac{1}{N} \sum_{i \in I_{3}} C_{U_{1} U_{2}}(i)>\frac{1-\mathbb{E}\{a\}}{N} \sum_{i \in I_{1}} C_{S U_{1}}(i), \\
\frac{1}{N} \sum_{i \in I_{3} \backslash \xi} C_{U_{1} U_{2}}(i)<\frac{1-\mathbb{E}\{a\}}{N} \sum_{i \in I_{1} \bigcup \xi} C_{S U_{1}}(i) .
\end{array}
$$

The above equations are hold if and only if moving subset $\xi$ from $I_{3}$ to $I_{1}$ creates discontinuity in $\frac{1-\mathbb{E}\{a\}}{N} \sum_{i \in I_{1}} C_{S U_{1}}(i)$ and $\frac{1}{N} \sum_{i \in I_{3}} C_{U_{1} U_{2}}(i)[5]$.

Since $\frac{|\xi|}{N} \rightarrow 0, \lim _{N \rightarrow \infty} \frac{1-\mathbb{E}\{a\}}{N} \sum_{i \in \xi} C_{S U_{1}}(i) \rightarrow 0$ and $\lim _{N \rightarrow \infty} \frac{1}{N} \sum_{i \in \xi} C_{U_{1} U_{2}}(i) \rightarrow 0$, it is impossible to cause discontinuity in $\frac{1-\mathrm{E}\{a\}}{N} \sum_{i \in I_{1}} C_{S U_{1}}(i)$ and $\frac{1}{N} \sum_{i \in I_{3}} C_{U_{1} U_{2}}(i)$. Hence, (31) is not correct and $\frac{1}{N} \sum_{i \in I_{3}} C_{U_{1} U_{2}}(i)=\bar{R}_{U_{1} U_{2}}=$ $(1-\mathbb{E}\{a\}) \bar{R}_{S U_{1}}=\frac{1-\mathbb{E}\{a\}}{N} \sum_{i \in I_{1}} C_{S U_{1}}(i)$ is hold. So, in the optimal link selection, $\bar{R}_{S U_{1}}-\bar{R}_{S U_{2}}=\bar{R}_{U_{1} U_{2}}$ and we have $\mathbb{E}\left\{b C_{U_{1} U_{2}}\right\}=\mathbb{E}\left\{(1-a)(1-b) C_{S U_{2}}\right\}$. This completes the proof.

\section{APPENDIX B}

According to the constraint $\mathrm{C}_{1}$, the average reception rate of user 2 is equal to $\frac{1}{N} \sum_{i=1}^{N}\left(1-b_{i}\right) C_{S U_{1}}(i)$. Thus, the Lagrangian function for the optimization problem in (13) can be written as

$$
\begin{gathered}
\mathcal{L}=\frac{1}{N} \sum_{i=1}^{N}\left(1-b_{i}\right) \log _{2}\left(1+S U_{1}(i)\right)-\frac{1}{N} \sum_{i=1}^{N} \alpha_{i} b_{i}\left(1-b_{i}\right) \\
-\frac{\mu}{N} \sum_{i=1}^{N}\left[b_{i} C_{U_{1} U_{2}}(i)-\left(1-a_{i}\right)\left(1-b_{i}\right) C_{S U_{1}}(i)\right]
\end{gathered}
$$

where $\mu$ and $\alpha_{i}$ are the Lagrange multipliers of constraints $\mathrm{C} 1$ and $\mathrm{C} 2$, respectively. By differentiating the Lagrangian function with respect to $b_{i}$ and equating the result to zero we obtain

$$
b_{i}=\frac{\left(1+\mu\left(1-a_{i}\right)\right) C_{S U_{1}}(i)+\mu C_{U_{1} U_{2}}(i)+\alpha_{i}}{2 \alpha_{i}} .
$$

Due to the existence of two choices for $b_{i}$, the variable $\alpha_{i}$ has also two possible values [5].

$$
\left\{\begin{array}{c}
b_{i}=0 \rightarrow \alpha_{i}^{1}=-\left(1+\mu\left(1-a_{i}\right)\right) C_{S U_{1}}(i)-\mu C_{U_{1} U_{2}}(i), \\
b_{i}=1 \rightarrow \alpha_{i}^{2}=\left(1+\mu\left(1-a_{i}\right)\right) C_{S U_{1}}(i)+\mu C_{U_{1} U_{2}}(i),
\end{array}\right.
$$

where $\alpha_{i}^{2}=-\alpha_{i}^{1}$. The Lagrangian function in (36) is maximized when $\alpha_{i}$ is negative. Thus, for $b_{i}$ we have

$$
\left\{\begin{array}{l}
b_{i}=0 \quad \text { if }\left(1+\mu\left(1-a_{i}\right)\right) C_{S U_{1}}(i)+\mu C_{U_{1} U_{2}}(i)>0, \\
b_{i}=1 \quad \text { if }\left(1+\mu\left(1-a_{i}\right)\right) C_{S U_{1}}(i)+\mu C_{U_{1} U_{2}}(i) \leq 0,
\end{array}\right.
$$

If we set $\lambda=\frac{1+\mu\left(1-a_{i}\right)}{-\mu}$, it is identical to (14). $\lambda$ or $\mu$ are chosen such that constraint $\mathrm{C}_{1}$ of problem (13) is satisfied. This completes the proof.

\section{REFERENCES}

[1] D. Meulen, "Three-Terminal Communication Channels," Advances in Applied Probability, vol. 3, no. 14, pp. 120-154, 1971.

[2] A. Host-Madsen and J. Zhang, "Capacity Bounds and Power Allocation for Wireless Relay Channels,' IEEE Transactions on Information Theory, vol. 51, no. 6, pp. 2020-2040, Jun. 2005.

[3] B. Xia, Y. Fan, J. Thompson and H. V. Poor, "Buffering in a Three-Node Relay Network," IEEE Transactions on Wireless Communications, vol. 7, no. 11, pp. 4492-4496, Nov. 2008.

[4] A. Ikhlef, D. S. Michalopoulos and R.Schober, "Max-Max Relay Selection for Relays with Buffers," IEEE Transactions on Wireless Communications, vol. 11, no. 3, pp. 1124-1135, March 2012.

[5] N. Zlatanov, R. Schober and P. Popovski,'Buffer-Aided Relaying with Adaptive Link Selection," IEEE Journal on Selected Areas in Communications, vol. PP, no. 99, pp. 1-13, August 2013.

[6] B. Maham and A. Hjrungnes, "Amplify-and-Forward Space-Time Coded Cooperation via Incremental Relaying," IEEE International Symposium on Wireless Communication Systems, pp. 407-411, Trondheim, Norway, Oct. 17-19, 2007.

[7] A. Ikhlef, D. S. Michalopoulos and R. Schober, "Buffers Improve The Performance of Relay Selection," IEEE Global Communication Conference, vol. 7, no. 8, pp. 1-6, Dec. 2011.

[8] I. Krikidis, Th. Charalambous and J. S. Thompson,'Buffer-Aided Relay Selection for Cooperative Diversity Systems without Delay Constraints,' IEEE Transactions on Wireless Communications, Vol. 11, no. 5, pp. 19581967, May 2012. 